\title{
Rational design of Two-dimensional Binary Polymers from Hetero Triangulenes for Photocatalytic Water Splitting
}

Zhenpei Zhou, ${ }^{\dagger}$ Maximilian A. Springer, ${ }^{\ddagger}{ }^{\S}$ Weixiang Geng, ${ }^{\dagger}$ Xinyue Zhu, ${ }^{\dagger}$ Tianchun $\mathrm{Li},{ }^{\dagger}$ Manman Li,${ }^{\dagger}$ Yu Jing, ${ }^{*, \dagger}$ and Thomas Heine ${ }^{*,+, \S}$

†Jiangsu Co-Innovation Centre of Efficient Processing and Utilization of Forest Resources, College of Chemical Engineering, Nanjing Forestry University, Nanjing 210037, China

\$TU Dresden, Fakultät Chemie und Lebensmittelchemie, Bergstraße 66c, 01062 Dresden, Germany

${ }^{\S}$ Helmholtz-Zentrum Dresden-Rossendorf, Institute of Resource Ecology, Permoserstraße 15, 04318 Leipzig, Germany

*email: yujing@njfu.edu.cn; thomas.heine@tu-dresden.de

\begin{abstract}
Based on first principles calculations, we report the design of three two-dimensional (2D) binary honeycomb-kagome polymers composed of B- and N-centered heterotriangulenes with a periodically alternate arrangement as in hexagonal boron nitride. The 2D binary polymers with donor-acceptor characteristics, are semiconductors with a direct band gap of 1.98-2.28 eV. The enhanced in-plane electron conjugation contributes to high charge carrier mobilities for both electrons and holes, about 6.70 and $0.24 \times 10^{3} \mathrm{~cm}^{2} \mathrm{~V}^{-1} \mathrm{~s}^{-1}$, respectively, for the 2D binary polymer with carbonyl bridges (2D CTPAB). With appropriate band edge alignment to match the water redox potentials and pronounced light adsorption for the ultraviolet and visible range of spectra, $2 \mathrm{D}$ CTPAB is predicted to be an effective photocatalyst/photoelectrocatalyst to promote overall water splitting.
\end{abstract}


TOC GRAPHICS

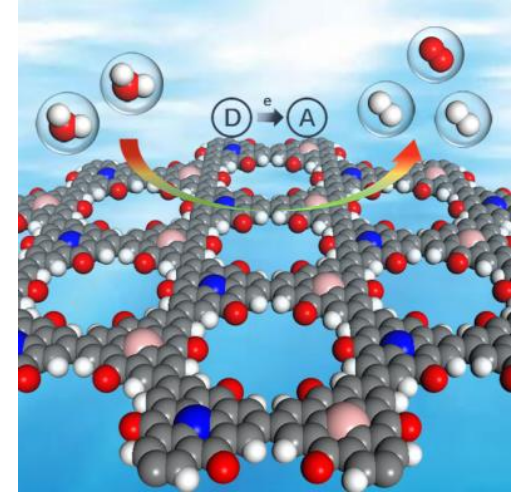

KEYWORDS two-dimensional materials; binary polymers; 2D polymers, heterotriangulenes; first-principles calculations; photocatalytic water splitting 
Fujishima and Honda demonstrated in $1972^{1}$ that water can be split into hydrogen and oxygen on rutile $\mathrm{TiO}_{2}$ using sunlight as the agent. This discovery launched the field of photocatalytic water splitting as promising strategy to produce green hydrogen. ${ }^{2,3,4}$ Since then, great efforts have been devoted to exploring effective photocatalysts, including transition metal oxides, ${ }^{5-8}$ nitrides ${ }^{9}$ and sulfides. ${ }^{10-12}$ However, few of them show satisfactory photocatalytic performance for water splitting because of the low quantum efficiency caused by charge recombination on both surface and bulk of the catalysts. ${ }^{13,14}$

The discovery of graphene in 2004 has greatly promoted the development of atomically thin two-dimensional (2D) materials. ${ }^{15-18}$ Once electrons and holes are generated under light irradiation, they can immediately participate in the on-surface hydrogen evolution reaction (HER) and oxygen evolution reaction (OER), respectively. Therefore, 2D semiconductors are considered as promising photocatalysts for water splitting ${ }^{19-21}$ and superior to bulk crystals where diffusion of the charge carriers to reach the surface results in high electron/hole recombination rates and limits performance. Aside from $2 \mathrm{D}$ transition metal-based compounds, ${ }^{22} 2 \mathrm{D}$ covalent organic polymers with moderate band gaps have attracted more and more attention, as their advantages include the absence of metals, high porosity, and a large manifold of geometrical topologies. ${ }^{23-26}$ However, despite the abundance of molecular monomers and the availability of new synthetic strategies, only a few of the known structures show satisfactory photocatalytic performance for water splitting. ${ }^{27-30}$ Among the most studied systems is $\mathrm{g}-\mathrm{C}_{3} \mathrm{~N}_{4}$, which shows suitable band structure ${ }^{31}$ but fails to overcome the high overpotential of OER, ${ }^{32}$ and co-catalysts or sacrificial agents are required to drive the water splitting reaction. ${ }^{33-35}$ Therefore, rationally designing 2D polymers with appropriate band structure for high catalytic activity for both HER and OER is still challenging and demanding.

Honeycomb-kagome 2D heterotriangulene (HT) polymers $^{36-39}$ are particularly promising because of their straightforward threefold symmetrical functionalization and high degree of $\pi$-conjugation. ${ }^{40,41}$ Our previous theoretical investigation indicated 
that B- or N-centered HTs based 2D polymers are single-band semiconductors with high carrier mobility for either exclusively electrons (B center) or holes ( $\mathrm{N}$ center), ${ }^{42}$ and the appropriate band edge alignment render them potential photocatalysts for either HER or OER processes, respectively, ${ }^{43}$ and coupling them in a tandem cell with spatially separate electrodes allows water splitting without the emergence of explosive $\mathrm{H}_{2} / \mathrm{O}_{2}$ mixture, yet at the expense of a 2-photon process per excited charge carrier.

An alternative route has been suggested for layered, so-called electron donor-acceptor covalent-organic frameworks, where electron-donating and -accepting building blocks are coupled in a bicontinuous manner. ${ }^{44,45}$ Such heterojunction is conducive to transfer photoinduced charge carriers between two half electrodes. ${ }^{46,47}$ Here, we theoretically demonstrate that the same capability can be achieved if the heterojunction is realized in a single plane as proposed in the title compounds, resulting in a single-material high quantum efficiency photocatalyst by coupling Band N-HTs into a honeycomb-kagome structure with alternating distribution of heteroatoms. We confirm their stability and photocatalytic activity by employing first principles calculations. The donor-acceptor coupling effect, band edge alignments, light absorbance and the carrier mobilities are rationalized by analyzing the band structure. Finally, the thermodynamic HER and OER processes are simulated to understand the potentials of applying these 2D polymers for effective solar-driven water splitting.

First-principles calculations on the basis of density functional theory (DFT) as implemented in the Vienna ab initio simulation package (VASP) ${ }^{48,49}$ were performed to optimize the hexagonal lattices of the $2 \mathrm{D}$ binary polymers. The projector augmented wave (PAW) method was used to treat the ion-electron interactions for atoms. ${ }^{50,51}$ The generalized gradient approximation (GGA) involving the Perdew-Burke-Ernzerhof (PBE) functional with Grimme's D3 van der Waals correction was adopted to process the exchange-correlation term and accurately account for the weak interactions between the adsorbents and the 2D binary 
polymers. ${ }^{52,53} \mathrm{~A}$ convergence threshold of $1 \times 10^{-5} \mathrm{eV}$ in energy and $1 \times 10^{-2} \mathrm{eV}^{-1}$ in force were set with the conjugate gradient method. A cutoff energy of $500 \mathrm{eV}$ for the plane wave basis set and the $3 \times 3 \times 1$ Monkhorst-Pack $k$-point mesh for the hexagonal Brillouin Zone was utilized in all computations. The vacuum layer was set to be $15 \AA$ along the $c$ direction to avoid interactions between neighboring images. Born-Oppenheimer molecular dynamics (BOMD) simulation controlled by using the Nosé-Hoover thermostat was performed at a temperature of $500 \mathrm{~K}$ for 5 ps to identify the thermal stability of the designed structures. The Heyd-Scuseria-Ernzerhof screened hybrid functional (HSE06) ${ }^{54}$ was employed to calibrate the band structures as the GGA-PBE functional usually underestimates the band gaps. The accuracy of HSE06 in reproducing the band structure of 2D HT-polymers has been verified in previous studies. ${ }^{40,42}$ In order to assess the ability of photocatalytic water splitting of 2D binary polymers, the band edges were corrected by the vacuum level and compared with the water redox potentials. Carrier mobilities were calculated using deformation potential theory (DPT). ${ }^{55}$ Detailed computational settings are given in supporting information.

\section{Geometry and formation energy of 2D binary HT-polymers}

Figure 1a shows the coupling of the B- and N-centered HTs and their arrangement into a 2D binary polymer. The designed structures differ only by the bridge functional groups (Figure $1 \mathrm{~b}-\mathrm{d}$ ) and are termed as carbonyl-bridged triphenylamine-triphenylborane (CTPAB), oxygen-bridged triphenylamine-triphenylborane (OTPAB) and dimethylene-bridged triphenylamine-triphenylborane (MTPAB), respectively. All the three structures show a planar configuration with single atomic layer thickness. The kagome-honeycomb $2 \mathrm{D}$ binary HT-polymers show a reduced $\mathrm{D}_{3 \mathrm{~h}}$ symmetry, analogous to that of h-BN. ${ }^{56}$ The optimized lattice parameters are found to be 17.47, 17.15 and $17.53 \AA$ for CTPAB, OTPAB and MTPAB, respectively, which are very similar and inbetween those of the unary B and N centered HT-polymers. More structural details including pore size and distance of two in-plane neighboring center atoms are also exhibited in 
Table S1 ${ }^{42}$ BOMD simulations (Figure $\mathrm{S} 1$ ) show that 2D binary HT-polymers remain intact at $500 \mathrm{~K}$ after 5 ps and recover the initial structure after subsequent geometry optimization, indicating the high thermal stability of the designed structures.

a
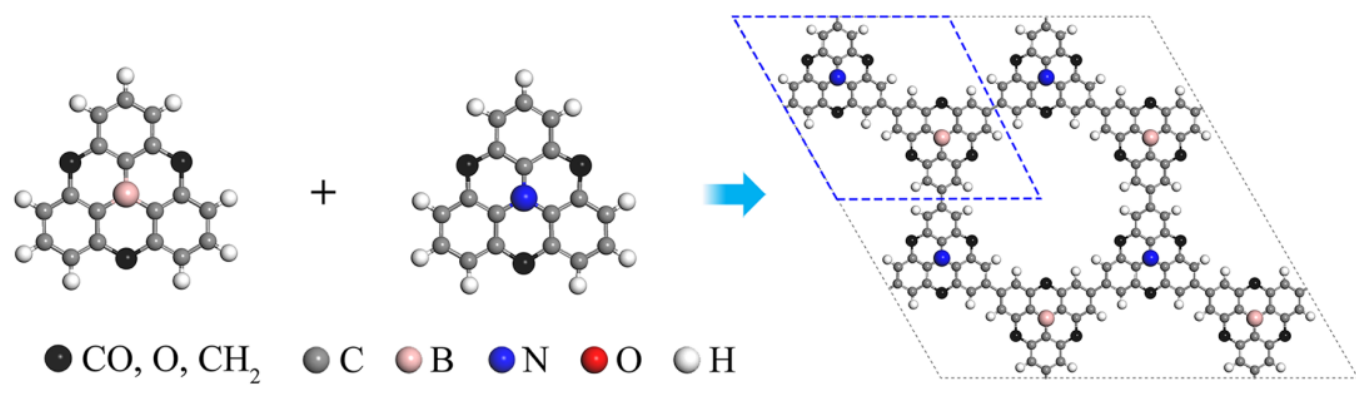

b

C

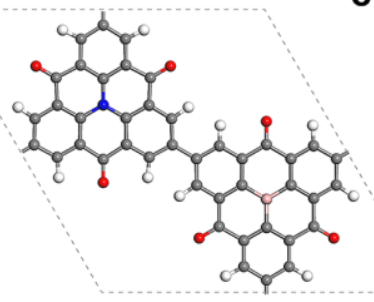

d
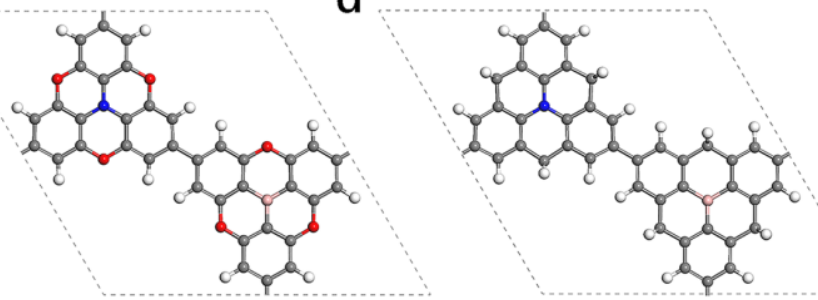

Figure 1. (a) Schematic view of the coupling of B- and N- centered HTs into a 2D binary polymer and the formed unit cells of CTPAB (b), OTPAB (c) and MTPAB (d).

The integrity of the alternating arrangement of $\mathrm{B}$ and $\mathrm{N}$ heteroatoms is confirmed by calculating the relative formation energy with respect to their monomers:

$$
E_{\mathrm{f}}=E_{\text {polymer }}+3 E_{\mathrm{H}_{2}}-E_{B \text {-monomer }}-E_{N \text {-monomer }}
$$

where $E_{\text {polymer, }} E_{B \text {-monomer }}$ and $E_{N \text {-monomer }}$ are the total energy of $2 \mathrm{D}$ binary polymers, the B- and N-centered HTs monomers, respectively, and $E_{\mathrm{H}_{2}}$ is the energy of the released hydrogen molecule. As presented in Table S1, the formation energies of 2D СТРAB, ОТРАВ, МТРАВ are found to be $1.56,1.59$ and $1.38 \mathrm{eV}$, respectively. For all three cases, these values are also more stable compared to the unary counterparts (e.g. 2E $\left.\mathrm{E}_{\text {CTPAB vs. }} \mathrm{E}_{\mathrm{CTPA}}+\mathrm{E}_{\mathrm{CTPB}}\right)$ by 91 (СТPAB), 51 (OTPAB) and 34 (MTPAB) meV per unitcell, respectively, and suggests the spontaneous formation of the binary polymers by the on-surface coupling of a mixture of B- and N-HTs monomers.

Electronic and optical properties of 2D binary HT-polymers

The band structures of 2D binary HT-polymers computed at the HSE06 level of 
theory are given in Figures 2a-c and reveal that all three 2D polymers are semiconductors with a direct band gap of $2.12 \mathrm{eV}, 1.98 \mathrm{eV}$ and $2.28 \mathrm{eV}$ for $2 \mathrm{D}$ CTPAB, OTPAB and MTPAB, respectively, smaller than that of the corresponding unary counterparts (Table S1). Both the conduction band minimum (CBM) and valence band maximum (VBM) of 2D binary HT-polymers are located at the K point. Compared to the single-band characteristics of the 2D unary HT-polymers, ${ }^{40-42}$ both the valence and conduction bands of 2D CTPAB, OTPAB and MTPAB near the Fermi level show pronouncedly dispersed bands due to the reduced symmetry from the unary counterparts. ${ }^{57}$ Since the HT-polymers are of honeycomb-kagome topology, the band structure around the Fermi level is dictated by the center atoms at the crystallographic positions $(1 / 3,1 / 3)$ and $(2 / 3,2 / 3)$. Therefore, the band structure can be coarse-grained by a tight-binding model (Equation S1) for the honeycomb network with two atoms per unit cell. In case both atoms have the same on-site energy, the band gap is similar to the well-known band structure of graphene, as shown in Figure $2 \mathrm{~d}$. However, if the on-site energies are not equal, which models the situation in $h$-BN, a band gap opens (Figure 2e). In this case, the band gap is of the size of the difference in on-site energy. It can be found that the band structures of the binary HT-polymers (Figure 2a-c) are similar to that of $h$-BN even though the boron and nitrogen centers are $1 \mathrm{~nm}$ apart from each other. This result is fully in line with our previous study, ${ }^{42}$ demonstrating the importance of lattice topology in controlling the electronic structures of HT-polymers. 

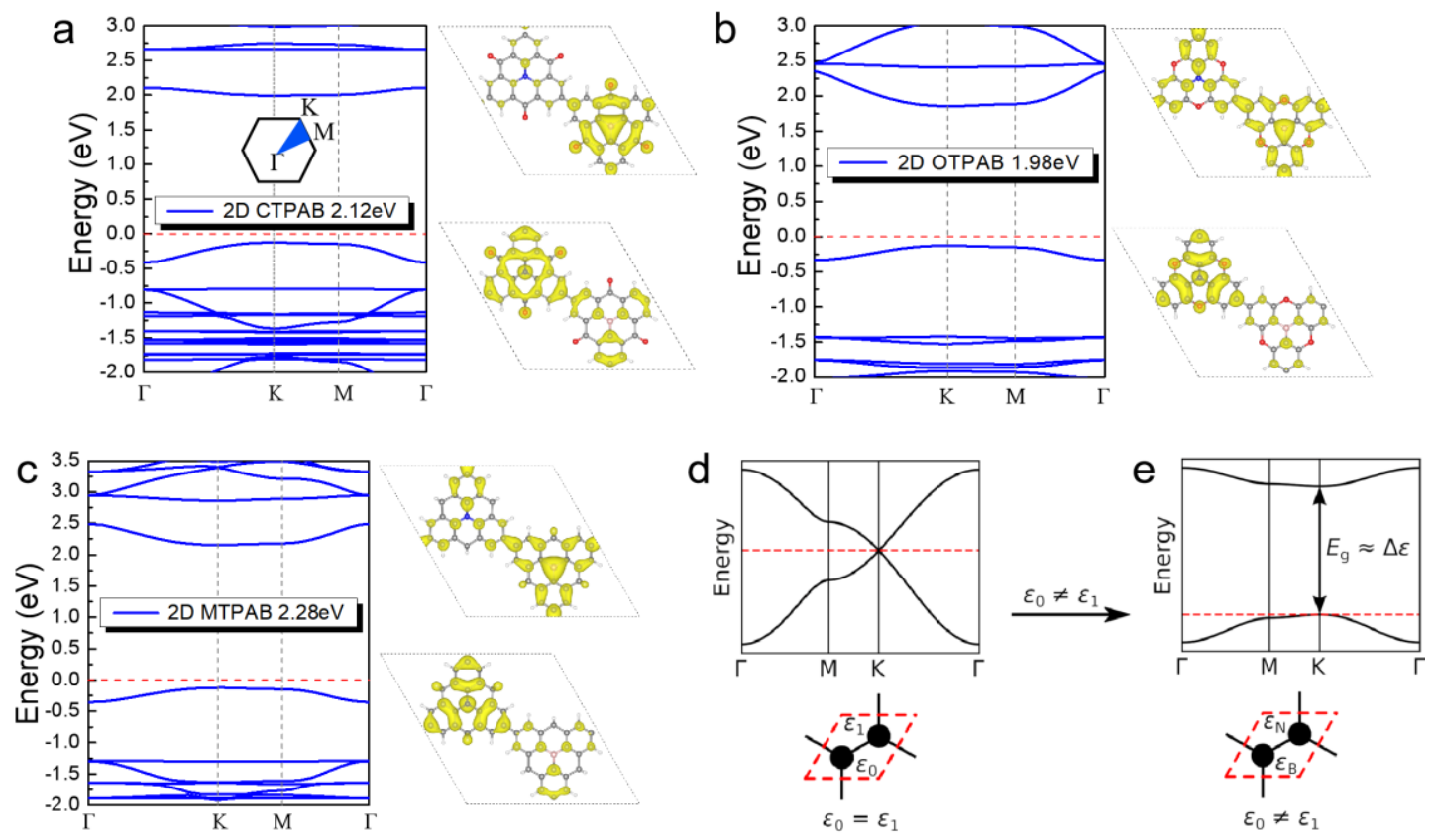

Figure 2. Band structures computed at the HSE06 level of theory, and corresponding partial charge density distribution for the CBM (above) and VBM (below), respectively, for (a) CTPAB, (b) OTPAB and (c) MTPAB. The isosurface for all orbitals was set to be $0.0013 \mathrm{e} \AA^{-3}$. The hexagonal Brillouin Zone is illustrated in the band structure of Figure 2a. (d) Band structure and sketch of a honeycomb network with equal on-site energies $\varepsilon_{0}$ and $\varepsilon_{1}$. The band structure is similar to the well-known graphene. (e) for non-equal on-site energies $\varepsilon_{0}=\varepsilon_{B}$ and $\varepsilon_{1}=\varepsilon_{N}$, being the on-site energies of boron and nitrogen, respectively, a band gap of the size of the difference in on-site energy opens (tight-binding parameters are given in the SI).

The partial charge density distribution analysis shows that the $\pi$ electron density is distributed along the entire skeleton of the binary polymers, indicating the effective $\pi$-conjugation in the $2 \mathrm{D}$ lattices. The chemical potential variation of $\mathrm{N}$ and $\mathrm{B}$ heteroatoms is evident by the analysis of CBM (dominated by B-centered monomer and VBM (dominated by N-centered monomer), indicating the $\mathrm{p}-\mathrm{n}$ type heterojunction characteristics. Bridge functionalization shapes the distinct valence and conduction band curvatures of the three structures, where CTPAB shows a more dispersed valence band, while OTPAB and MTPAB show a more dispersed conduction band. The high charge fluctuation affects the in-plane p-n type characteristics and thus the charge transport properties that distinguish the 2D HTs 
binary polymers from their unary counterparts.

Table 1. In-plane stiffness $\left(C_{2 \mathrm{D}}\right)$, DP constant $\left(\left|E_{1}\right|\right)$, effective mass $\left(\left|m^{*}\right|\right)$ of electrons and holes, and carrier mobilities $(\mu)$ for 2D binary polymers along the armchair direction. The carrier mobilities were calculated at the PBE level of theory and $m_{0}$ indicates the mass of a single electron.

\begin{tabular}{|c|c|c|c|c|}
\hline $\begin{array}{l}\text { 2D binary } \\
\text { polymers }\end{array}$ & $\mathrm{C}_{2 \mathrm{D}} / \mathrm{N} \mathrm{m}^{-1}$ & $\left|E_{1}\right| / \mathrm{eV}$ & $\left|m^{*}\right| / m_{0}$ & $\begin{array}{c}\mu / \times 10^{3} \mathrm{~cm}^{2} \mathrm{~V}^{-1} \\
\mathrm{~s}^{-1}\end{array}$ \\
\hline \multirow{3}{*}{ СТРАВ } & & 0.21 & $1.71\left(m_{\mathrm{e}}\right)$ & 6.70 \\
\hline & 59.60 & & & \\
\hline & & 2.15 & $0.87\left(m_{\mathrm{h}}\right)$ & 0.24 \\
\hline \multirow{3}{*}{ ОТРАВ } & & 2.58 & $0.61\left(m_{\mathrm{e}}\right)$ & 0.37 \\
\hline & 64.23 & & & \\
\hline & & 3.59 & $0.90\left(m_{\mathrm{h}}\right)$ & 0.087 \\
\hline \multirow{3}{*}{ МТРАВ } & & 2.44 & $0.68\left(m_{\mathrm{e}}\right)$ & 0.32 \\
\hline & 62.07 & & & \\
\hline & & 3.23 & $0.83\left(m_{\mathrm{h}}\right)$ & 0.12 \\
\hline
\end{tabular}

Details on the calculated carrier mobilities are given in Figure S2 and Table S2. 2D binary HT-polymers present effective charge transport for both electrons and holes (Table 1), with significant tuning by the bridge functionalizations. Specifically, the $\pi$-electron density of the $2 \mathrm{D}$ skeleton is more distributed at the $\mathrm{C}=\mathrm{O}$ bridges of CTPAB than that at the $\mathrm{CH}_{2}$ and $\mathrm{O}$ brides of MTPAB and OTPAB, respectively. As a result, the structure deformation are less impactive to the wave functions of valence and conduction bands of CTPAB, resulting in smaller DP constants and higher carrier mobilities. The carrier mobility of 2D CTPAB is as high as 6.70 and $0.24 \times 10^{3} \mathrm{~cm}^{2}$ $\mathrm{V}^{-1} \mathrm{~s}^{-1}$ for electrons and holes respectively, values comparable to those of crystalline silicon $\left(\sim 1.40 \times 10^{3} \mathrm{~cm}^{2} \mathrm{~V}^{-1} \mathrm{~s}^{-1}\right),{ }^{58}$ making this material besides a good photocatalyst candidate for water splitting also a promising 2D organic electronics material.

The first step to evaluate photocatalytic activity is to compare the band edge 
alignments with the water redox potentials. Generally, to facilitate overall water splitting, the VBM of the photocatalyst must be lower than the OER potential (-5.67 $\mathrm{eV}$ vs. vacuum), while the CBM is required to locate above the HER potential (-4.44 eV vs. vacuum). As illustrated in Figure 3a, 2D CTPAB shows appropriate band edge alignments for overall water splitting, while $2 \mathrm{D}$ OTPAB and MTPAB only meet the requirement for the HER process. The band edge distribution tendency of these binary HT-polymers is identical to their corresponding unary counterparts, indicating again the importance of appropriate bridge functionalization.

Since the binary HT-polymers show reduced band gap compared to their unary counterparts, enhanced light harvesting capability can be expected, which is confirmed by our prediction shown in Figure $3 \mathrm{~b}$ for all three candidate structures, both for the ultraviolet and visible spectra, with an absorption coefficient of up to $10^{5}$ $\mathrm{cm}^{-1}$, comparable to organic perovskite solar cells. ${ }^{59}$
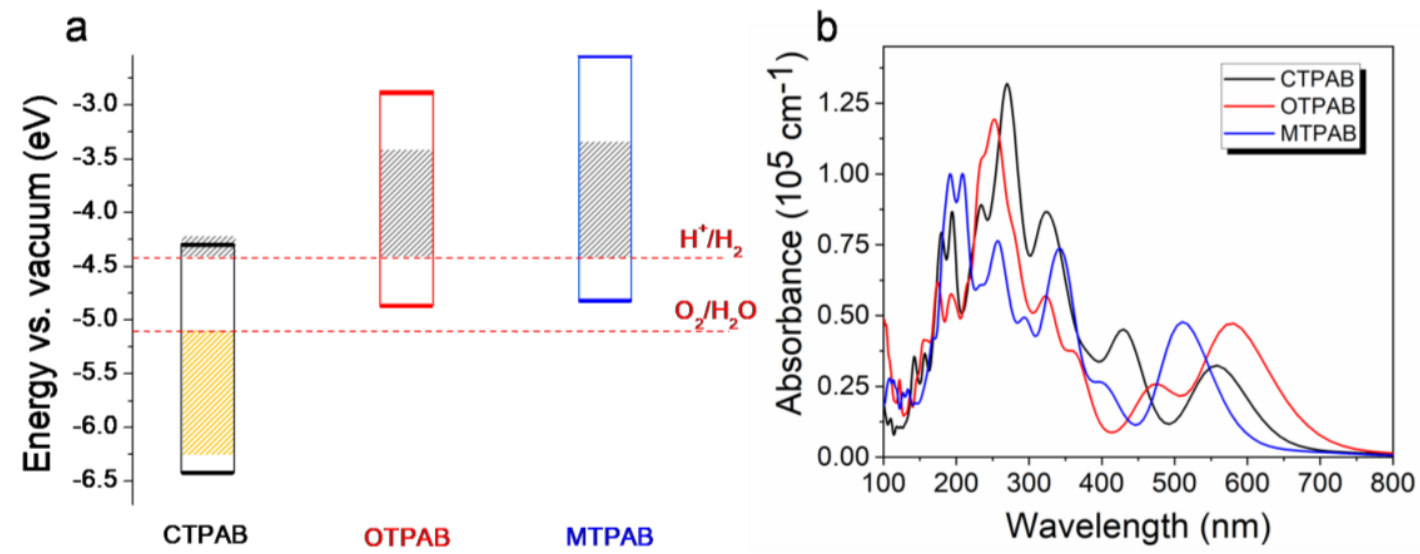

Figure 3. (a) The band edges of $2 \mathrm{D}$ binary HT-polymers in comparison with the water redox potentials at $\mathrm{pH}=0$ (indicated by the red dashed lines). The original overpotential of HER ( $\eta_{\text {HER }}$ ) on CTPAB, OTPAB and MTPAB and the OP of OER ( shaded bars. (b) Calculated absorption spectra of the three binary HT-polymers for light induced from the in-plane directions. 
Finally, we studied the specific thermodynamic processes of HER and OER on their surfaces. The HER process is accompanied by the formation of adsorbed $\mathrm{H}^{*}$ intermediate. All possible $\mathrm{H}^{*}$ adsorption configurations have been examined. By comparing the adsorption energy of $\mathrm{H}^{*}$ on different potential active sites, we found that the $\mathrm{H}^{*}$ species tends to be adsorbed on the $\mathrm{O}$ site at the bridge (Figure 4a) of the B-monomer side for 2D CTPAB but the carbon sites of the B-monomer side for 2D OTPAB and MTPAB (Figures S2 a and b). By examining the Gibbs free energy change $(\Delta G)$ of the elementary steps of HER without applying external potential, we found that the HER process on 2D CTPAB, OTPAB and MTPAB is unfavorable by showing a positive $\Delta G$ value of $0.21,1.03$ and $1.12 \mathrm{eV}$ for the formation of $\mathrm{H}^{*}$, respectively, contributing to an uphill free energy profile of the first HER step as indicated in Figure $4 \mathrm{~b}$. The $\eta_{\text {HER }}$ is calculated to be $0.21,1.03$ and $1.12 \mathrm{~V}$ for CTPAB, OTPAB and MTPAB (Equation S17), respectively (details can be found in the SI). Obviously, CTPAB is more active for HER than the other two structures due to the high electron affinity of $\mathrm{O}$ of the $\mathrm{C}=\mathrm{O}$ bridge. Upon light irradiation, the photogenerated electrons on CTPAB, OTPAB and MTPAB will contribute to a drive potential $\left(U_{\mathrm{e}}\right)$ of $0.14,1.55$ and $1.90 \mathrm{eV}$ for HER, respectively (Figure 3a). In this case, the HER process on OTPAB and MTPAB will proceed spontaneously with all the elementary steps becoming downhill. For CTPAB, although the first step of HER is still uphill upon irradiation, the remaining energy barrier is as negligible as $0.07 \mathrm{eV}$, which can be easily overcome by adding co-catalysts (e.g. Pt $)^{343434}$ or applying external electric potential. Note that the solvation effect is not considered in this work which may quantitatively influence the calculated Gibbs free energy of different intermediates. ${ }^{60}$ However, the differences in photocatalytic activity as a function of the composition of the building monomers will not be qualitatively affected by the presence of water solvent.

The OER process is more complicated and is accompanied by the gradual formation of oxygen-containing intermediates in a $4 \mathrm{e}$ process. Since $2 \mathrm{D}$ OTPAB and 
MTPAB are excluded from possible OER photocatalysts due to the unsuitable band edges, we only consider the OER process on 2D CTPAB here. As expected, 2D CTPAB inherits the catalytic activity from their unary counterparts, and the B-center is found to be the most favorable site for OER (Figure 4a). The free energy profiles corresponding to the formation of $\mathrm{OH}^{*}, \mathrm{O}^{*}$ and $\mathrm{OOH}^{*}$ species are first outlined in the absence of light irradiation. As shown in Figure 4c, all the four elementary steps are endothermic with the $\Delta G$ value of $1.60,1.84,1.14$ and $0.34 \mathrm{eV}$ for each step, indicating that the OER process cannot proceed on 2D CTPAB without an external potential. The rate determining step is the second oxidation step, corresponding to the formation of $\mathrm{O}^{*}$. The $\eta_{\mathrm{OER}}$ is calculated to be $0.61 \mathrm{~V}$ according to Equation S18. Under light irradiation, the photogenerated holes can provide an external drive potential of $0.75 \mathrm{~V}$ which can overcome the overpotential and contribute to a negative $\Delta G$ value for all the elementary steps. The dual-site process of OER is also considered as a comparison. As shown in Figure S3, the ךOER of the dual-site process remains to be $0.65 \mathrm{~V}$ under light irradiation, indicating that OER in this path is unfavorable. As a result, the OER process will spontaneously proceed in a single-site process on $2 \mathrm{D}$ CTPAB under light irradiation.

CTPAB can facilitate both HER and OER processes on different surface active sites and the donor-acceptor characteristics is beneficial to effective electron/hole separation and will contribute to high quantum efficiency. However, co-catalysts or external electric potential are necessary to overcome the small energy barrier of HER under light-irradiation. Therefore, $2 \mathrm{D}$ CTPAB is predicted to be a promising non-metal photocatalyst or photoelectrocatalyst to facilitate overall water splitting. 

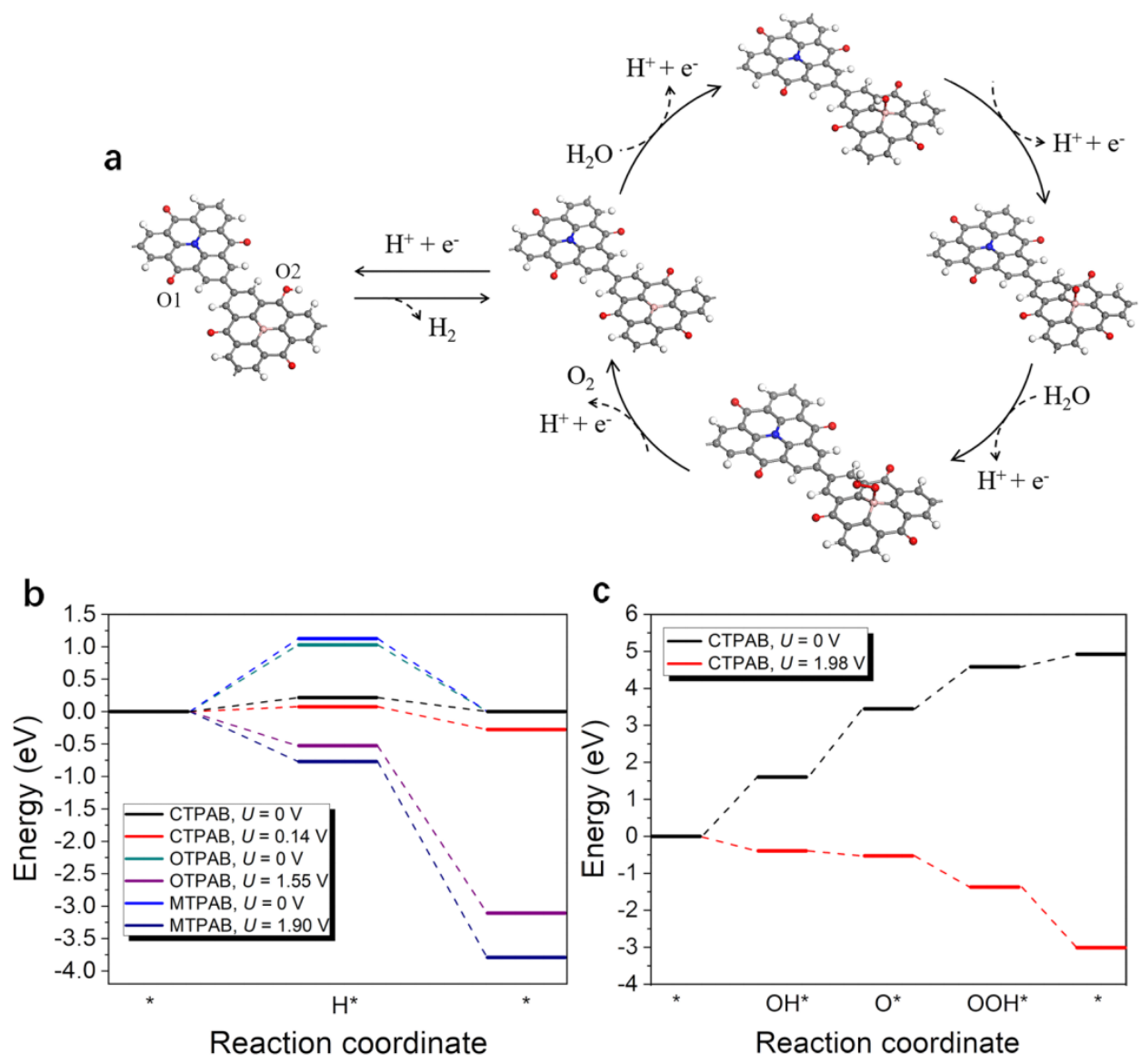

Figure 4. (a) Schematics for the elementary steps of HER and OER on CTPAB; Free energy profiles of HER on three 2D binary polymers (b) and OER on CTPAB (c) in the absence of light irradiation $(U=0 \mathrm{~V})$ and under light irradiation $\left(U=U_{\mathrm{e}}\right.$ or $\left.U_{\mathrm{h}}\right)$.

To summarize, by means of first principles calculations, we have systematically studied the structural, electronic and optical properties of three 2D binary polymers that are made of B- and N- centered HTs and explored their photocatalytic activity for water splitting. The integration of $\mathrm{B}$ - and $\mathrm{N}$ - centered HTs into a monolayer contributes to the formation of donor-acceptor type 2D networks, which are semiconductors with a direct band gap of 1.98-2.28 eV. The more narrowed band gap and higher dispersion of valence and conduction bands of these 2D binary HT-polymers than that of their unary counterparts, contribute to higher charge carrier mobility and more pronounced light absorption in the visible range of spectrum. With appropriate band edges and sufficient drive potential for both HER and OER, 2D CTPAB is predicted to be a promising photocatalyst/photoelectrocatalyst for overall 
water splitting. Our investigations demonstrate that lattice topology and component optimization including center atoms and bridge functional groups play an important role in controlling the electronic and optical properties of 2D polymers, which pave a new way in designing promising non-metal photocatalysts for overall water splitting.

\section{ASSOCIATED CONTENT}

\section{Supporting Information.}

The Supporting Information is available free of charge on the ACS Publications website, including the description of the geometry and electronic properties of $2 \mathrm{D}$ binary HT-polymers and computational details of the carrier mobilities and thermodynamics of oxygen and hydrogen evolution reactions.

\section{AUTHOR INFORMATION}

\section{Notes}

The authors declare no competing financial interests.

\section{ACKNOWLEDGEMENTS}

The work obtained financial support in China by the National Natural Science Foundation of China (no. 21903046) and the NSF of Jiangsu Province of China (no. BK20190744). The computational resources utilized in this research were provided by Shanghai Supercomputer Center. CRC 1415 and ZIH Dresden are thanked for their continuous infrastructural support. This work is dedicated to the 100th anniversary of Chemistry at Nankai University 


\section{REFERENCES}

(1) Fujishima, A.; Honda, K. Electrochemical Photolysis of Water at a Semiconductor Electrode. Nature 1972, 238, 37-38.

(2) Nozik, A. J. Photoelectrolysis of water using semiconducting $\mathrm{TiO}_{2}$ crystals. Nature 1975, 257, 383-386.

(3) Takata, T.; Domen, K. Particulate Photocatalysts for Water Splitting: Recent Advances and Future Prospects. ACS Energy Lett. 2019, 4, 542-549.

(4) Rahman, M. Z.; Kibria, M. G.; Mullins, C. B. Metal-free photocatalysts for hydrogen evolution. Chem. Soc. Rev. 2020, 49, 1887-1931.

(5) Suntivich, J.; May, K. J.; Gasteiger, H. A.; Goodenough, J. B.; Shao-Horn, Y. A Perovskite Oxide Optimized for Oxygen Evolution Catalysis from Molecular Orbital Principles. Science 2011, $334,1383-1385$.

(6) Asahi, R.; Morikawa, T.; Ohwaki, T.; Aoki, K.; Taga, Y. Visible-Light Photocatalysis in Nitrogen-Doped Titanium Oxides. Science 2001, 293, 269-271.

(7) Yanagida, T.; Sakata, Y.; Imamura, H. Photocatalytic Decomposition of $\mathrm{H}_{2} \mathrm{O}$ into $\mathrm{H}_{2}$ and $\mathrm{O}_{2}$ over $\mathrm{Ga}_{2} \mathrm{O}_{3}$ Loaded with NiO. Chem. Lett. 2004, 33, 726-727.

(8) Maeda, K.; Takata, T.; Hara, M.; Saito, N.; Inoue, Y.; Kobayashi, H.; Domen, K. GaN:ZnO Solid Solution as a Photocatalyst for Visible-Light-Driven Overall Water Splitting. J. Am. Chem. Soc. 2005, 127, 8286-8287.

(9) Han, N.; Liu, P.; Jiang, J.; Ai, L.; Shao, Z.; Liu, S. Recent advances in nanostructured metal nitrides for water splitting. J. Mater. Chem. A 2018, 6, 19912-19933.

(10) Sun, S.; Hisatomi, T.; Wang, Q.; Chen, S.; Ma, G.; Liu, J.; Nandy, S.; Minegishi, T.; Katayama, M.; Domen, K. Efficient Redox-Mediator-Free Z-Scheme Water Splitting Employing Oxysulfide Photocatalysts under Visible Light. ACS. Catal. 2018, 8, 1690-1696.

(11) Ma, G.; Chen, S.; Kuang, Y.; Akiyama, S.; Hisatomi, T.; Nakabayashi, M.; Shibata, N.; Katayama, M.; Minegishi, T.; Domen, K. Visible Light-Driven Z-Scheme Water Splitting Using Oxysulfide $\mathrm{H}_{2}$ Evolution Photocatalysts. J. Phys. Chem. Lett. 2016, 7, 3892-3896.

(12) Tsuji, I.; Kato, H.; Kudo, A. Visible-light-induced $\mathrm{H}_{2}$ evolution from an aqueous solution 
containing sulfide and sulfite over a $\mathrm{ZnS}-\mathrm{CuInS}_{2}-\mathrm{AgInS} \mathrm{S}_{2}$ solid-solution photocatalyst. Angew.

Chem. Int. Ed. 2005, 44, 3565-3568.

(13) Fujishima, A.; Rao, T. N.; Tryk, D. A. Titanium dioxide photocatalysis. J. Photochem.

Photobiol., C 2000, 1, 1-21.

(14) Grätzel, M. Photoelectrochemical cells. Nature 2001, 414, 338-344.

(15) Novoselov, K. S.; Geim, A. K.; Morozov, S. V.; Jiang, D.; Zhang, Y.; Dubonos, S. V.; Grigorieva, I. V.; Firsov, A. A. Electric Field Effect in Atomically Thin Carbon Films. Science 2004, 306, 666-669.

(16) Novoselov, K. S.; Fal'ko, V. I.; Colombo, L.; Gellert, P. R.; Schwab, M. G.; Kim, K. A roadmap for graphene. Nature 2012, 490, 192-200.

(17) Heine, T. Transition metal chalcogenides: ultrathin inorganic materials with tunable electronic properties. Acc. Chem. Res. 2015, 48, 65-72.

(18) Wang, Y.; Li, Y.; Chen, Z. Planar Hypercoordinate Motifs in Two-Dimensional Materials. Acc. Chem. Res. 2020, 53, 887-895.

(19) Ju, L.; Shang, J.; Tang, X.; Kou, L. Tunable Photocatalytic Water Splitting by the Ferroelectric Switch in a $2 \mathrm{D} \mathrm{AgBiP}{ }_{2} \mathrm{Se}_{6}$ Monolayer. J. Am. Chem. Soc. 2020, 142, 1492-1500.

(20) Peng, Q.; Xiong, R.; Sa, B.; Zhou, J.; Wen, C.; Wu, B.; Anpo, M.; Sun, Z. Computational mining of photocatalysts for water splitting hydrogen production: two-dimensional InSe-family monolayers. Catal. Sci. Technol. 2017, 7, 2744-2752.

(21) Singh, A. K.; Mathew, K.; Zhuang, H. L.; Hennig, R. G. Computational Screening of 2D Materials for Photocatalysis. J. Phys. Chem. Lett. 2015, 6, 1087-1098.

(22) Qiao, M.; Liu, J.; Wang, Y.; Li, Y.; Chen, Z. $\mathrm{PdSeO}_{3}$ Monolayer: Promising Inorganic 2D Photocatalyst for Direct Overall Water Splitting Without Using Sacrificial Reagents and Cocatalysts. J. Am. Chem. Soc. 2018, 140, 12256-12262.

(23) Banerjee, T.; Gottschling, K.; Savasci, G.; Ochsenfeld, C.; Lotsch, B. V. $\mathrm{H}_{2}$ Evolution with Covalent Organic Framework Photocatalysts. ACS Energy Lett. 2018, 3, 400-409.

(24) Vyas, V. S.; Haase, F.; Stegbauer, L.; Savasci, G.; Podjaski, F.; Ochsenfeld, C.; Lotsch, B. V. A tunable azine covalent organic framework platform for visible light-induced hydrogen 
generation. Nat. Commun. 2015, 6, 8508 .

(25) Jin, E.; Geng, K.; Lee, K. H.; Jiang, W.; Li, J.; Jiang, Q.; Irle, S.; Jiang, D.

Topology-Templated Synthesis of Crystalline Porous Covalent Organic Frameworks. Angew.

Chem. Int. Ed. 2020, 59, 12162-12169.

(26) Springer, M. A.; Liu, T.-J.; Kuc, A.; Heine, T. Topological Two-dimensional Polymers. Chem. Soc. Rev. 2020, 49, 2007-2019.

(27) Jin, E.; Lan, Z.; Jiang, Q.; Geng, K.; Li, G.; Wang, X.; Jiang, D. 2D sp2 Carbon-Conjugated Covalent Organic Frameworks for Photocatalytic Hydrogen Production from Water. Chem 2019, $5,1632-1647$.

(28) Wang, Y.; Vogel, A.; Sachs, M.; Sprick, R. S.; Wilbraham, L.; Moniz, S. J. A.; Godin, R.; Zwijnenburg, M. A.; Durrant, J. R.; Cooper, A. I.; Tang, J. Current understanding and challenges of solar-driven hydrogen generation using polymeric photocatalysts. Nat. Energy 2019, 4, 746-760.

(29) Wang, L.; Wan, Y.; Ding, Y.; Wu, S.; Zhang, Y.; Zhang, X.; Zhang, G.; Xiong, Y.; Wu, X.; Yang, J.; $\mathrm{Xu}, \mathrm{H}$. Conjugated microporous polymer nanosheets for overall water splitting using visible light. Adv. Mater. 2017, 29, 1702428.

(30) Wan, Y.; Wang, L.; Xu, H.; Wu, X.; Yang, J. A simple molecular design strategy for two-dimensional covalent organic framework capable of visible-light-driven water splitting. J. Am. Chem. Soc. 2020, 142, 4508-4516.

(31) Wang, X.; Maeda, K.; Thomas, A.; Takanabe, K.; Xin, G.; Carlsson, J. M.; Domen, K.; Antonietti, M. A metal-free polymeric photocatalyst for hydrogen production from water under visible light. Nat. Mater. 2009, 8, 76-80.

(32) Valdés, Á.; Qu, Z.-W.; Kroes, G.-J.; Rossmeisl, J.; Nørskov, J. K. Oxidation and Photo-Oxidation of Water on $\mathrm{TiO}_{2}$ Surface. J. Phys. Chem. C 2008, 112, 9872-9879.

(33) Pan, Z.; Zheng, Y.; Guo, F.; Niu, P.; Wang, X. Decorating CoP and Pt Nanoparticles on Graphitic Carbon Nitride Nanosheets to Promote Overall Water Splitting by Conjugated Polymers. ChemSusChem 2017, 10, 87-90.

(34) Zhang, G.; Lan, Z. A.; Lin, L.; Lin, S.; Wang, X. Overall water splitting by Pt/g-C $\mathrm{C}_{3} \mathrm{~N}_{4}$ photocatalysts without using sacrificial agents. Chem. Sci. 2016, 7, 3062-3066.

(35) Zhang, G.; Lan, Z. A.; Wang, X. Surface engineering of graphitic carbon nitride polymers with cocatalysts for photocatalytic overall water splitting. Chem. Sci. 2017, 8, 5261-5274. 
(36) Kitamoto, Y.; Suzuki, T.; Miyata, Y.; Kita, H.; Funaki, K.; Oi, S. The first synthesis and X-ray crystallographic analysis of an oxygen-bridged planarized triphenylborane. Chem. Commun. 2016, 52, 7098-7101.

(37) Kushida, T.; Yamaguchi, S. A Radical Anion of Structurally Constrained Triphenylborane. Organometallics 2013, 32, 6654-6657.

(38) Schlütter, F.; Rossel, F.; Kivala, M.; Enkelmann, V.; Gisselbrecht, J. P.; Ruffieux, P.; Fasel, R.; Müllen, K. $\pi$-Conjugated heterotriangulene macrocycles by solution and surface-supported synthesis toward honeycomb networks. J. Am. Chem. Soc. 2013, 135, 4550-4557.

(39) Bieri, M.; Blankenburg, S.; Kivala, M.; Pignedoli, C. A.; Ruffieux, P.; Müllen, K.; Fasel, R. Surface-supported 2D heterotriangulene polymers. Chem. Commun. 2011, 47, 10239-10241.

(40) Steiner, C.; Gebhardt, J.; Ammon, M.; Yang, Z.; Heidenreich, A.; Hammer, N.; Görling, A.; Kivala, M.; Maier, S. Hierarchical on-surface synthesis and electronic structure of carbonyl-functionalized one- and two-dimensional covalent nanoarchitectures. Nat. Commun. 2017, 8, 14765.

(41) Galeotti, G.; De Marchi, F.; Hamzehpoor, E.; MacLean, O.; Rajeswara Rao, M.; Chen, Y.; Besteiro, L. V.; Dettmann, D.; Ferrari, L.; Frezza, F.; Sheverdyaeva, P. M.; Liu, R.; Kundu, A. K.; Moras, P.; Ebrahimi, M.; Gallagher, M. C.; Rosei, F.; Perepichka, D. F.; Contini, G. Synthesis of mesoscale ordered two-dimensional $\pi$-conjugated polymers with semiconducting properties. Nat. Mater. 2020, 19, 874-880.

(42) Jing, Y.; Heine, T. Two-dimensional Kagome Lattices Made of Hetero Triangulenes are Dirac Semimetals or Single-Band Semiconductors. J. Am. Chem. Soc. 2019, 141, 743-747.

(43) Jing, Y.; Zhou, Z.; Geng, W.; Zhu, X.; Heine, T. Two-Dimensional Honeycomb-Kagome Polymers as Effective Metal-Free Photocatalysts for Water Splitting. Adv. Mater. 2021, 33, 2008645.

(44) Chen, L.; Furukawa, K.; Gao, J.; Nagai, A.; Nakamura, T.; Dong, Y.; Jiang, D. Photoelectric Covalent Organic Frameworks: Converting Open Lattices into Ordered Donor-Acceptor Heterojunctions. J. Am. Chem. Soc. 2014, 136, 9806-9809.

(45) Jin, S.; Supur, M.; Addicoat, M.; Furukawa, K.; Chen, L.; Nakamura, T.; Fukuzumi, S.; Irle, S.; Jiang, D. Creation of Superheterojunction Polymers via Direct Polycondensation: Segregated and Bicontinuous Donor-Acceptor $\pi$-Columnar Arrays in Covalent Organic Frameworks for Long-Lived Charge Separation. J. Am. Chem. Soc. 2015, 137, 7817-7827. 
(46) Jin, S.; Ding, X.; Feng, X.; Supur, M.; Furukawa, K.; Takahashi, S.; Addicoat, M.; El-Khouly, M. E.; Nakamura, T.; Irle, S.; Fukuzumi, S.; Nagai, A.; Jiang, D. Charge Dynamics in A DonorAcceptor Covalent Organic Framework with Periodically Ordered Bicontinuous Heterojunctions. Angew. Chem. Int. Ed. 2013, 52, 2017-2021.

(47) Feng, X.; Chen, L.; Honsho, Y.; Saengsawang, O.; Liu, L.; Wang, L.; Saeki, A.; Irle, S.; Seki, S.; Dong, Y.; Jiang, D. An Ambipolar Conducting Covalent Organic Framework with Self-Sorted and Periodic Electron Donor-Acceptor Ordering. Adv. Mater. 2012, 24, 3026-3031.

(48) Kresse, G.; Hafner, J. Norm-conserving and ultrasoft pseudopotentials for first-row and transition elements. J. Phys.: Condens. Matter 1994, 6, 8245-8257.

(49) Sun, G.; Kürti, J.; Rajczy, P.; Kertesz, M.; Hafner, J.; Kresse, G. Performance of the Vienna ab initio simulation package (VASP) in chemical applications. J. Mol. Struct.: THEOCHEM 2003, $624,37-45$.

(50) Blöchl, P. E. Projector augmented-wave method. Phys. Rev. B 1994, 50, 17953-17979.

(51) Kresse, G.; Joubert, D. From ultrasoft pseudopotentials to the projector augmented-wave method. Phys. Rev. B 1999, 59, 1758-1775.

(52) Perdew, J. P.; Burke, K.; Ernzerhof, M. Generalized Gradient Approximation Made Simple. Phys. Rev. Lett. 1996, 77, 3865-3868.

(53) Grimme, S. Semiempirical GGA-type density functional constructed with a long-range dispersion correction. J. Comput. Chem. 2006, 27, 1787-1799.

(54) Heyd, J.; Peralta, J. E.; Scuseria, G. E.; Martin, R. L. Energy band gaps and lattice parameters evaluated with the Heyd-Scuseria-Ernzerhof screened hybrid functional. J. Chem. Phys. 2005, 123, 174101.

(55) Bardeen, J.; Shockley, W. Deformation Potentials and Mobilities in Non-Polar Crystals. Phys. Rev. 1950, 80, 72-80.

(56) Warner, J. H.; Rümmeli, M. H.; Bachmatiuk, A.; Büchner, B. Atomic Resolution Imaging and Topography of Boron Nitride Sheets Produced by Chemical Exfoliation. ACS Nano 2010, 4, 1299-1304.

(57) Thomas, S.; Li, H.; Zhong, C.; Matsumoto, M.; Dichtel, W. R.; Bredas, J.-L. Electronic Structure of Two-Dimensional $\pi$-Conjugated Covalent Organic Frameworks. Chem. Mater. 2019, $31,3051-3065$

(58) Jacboni, C.; Canali, C.; Otiaviani, G.; Quaranta, A. A. A Review of Some Charge Transport 
Properties of Silicon. Solid-State Electron. 1977, 20, 77-89.

(59) Shirayama, M.; Kadowaki, H.; Miyadera, T.; Sugita, T.; Tamakoshi, M.; Kato, M.; Fujiseki, T.; Murata, D.; Hara, S.; Murakami, T. N.; Fujimoto, S.; Chikamatsu, M.; Fujiwara, H. Optical Transitions in Hybrid Perovskite Solar Cells: Ellipsometry, Density Functional Theory, and Quantum Efficiency Analyses for $\mathrm{CH}_{3} \mathrm{NH}_{3} \mathrm{PbI}_{3}$. Phys. Rev. Appl. 2016, 5, 014012.

(60) Gauthier, J. A.; Dickens, C. F.; Chen, L. D.; Doyle, A. D.; Nørskov, J. K. Solvation effects for oxygen evolution reaction catalysis on $\mathrm{IrO}_{2}(110)$. J. Phys. Chem. C 2017, 121, 11455-11463. 